Network Working Group

Request for Comments: 2076

Category: Informational
J. Palme

Stockholm University/KTH

February 1997

\title{
Common Internet Message Headers
}

Status of this Memo

This memo provides information for the Internet community. This memo does not specify an Internet standard of any kind. Distribution of this memo is unlimited.

Abstract

This memo contains a table of commonly occurring headers in headings of e-mail messages. The document compiles information from other RFCs such as RFC 822, RFC 1036, RFC 1123, RFC 1327, RFC 1496, RFC 1521, RFC 1766, RFC 1806, RFC 1864 and RFC 1911. A few commonly occurring headers which are not defined in RFCs are also included. For each header, the memo gives a short description and a reference to the RFC in which the header is defined.

Table of contents

1. Introduction............................ 2

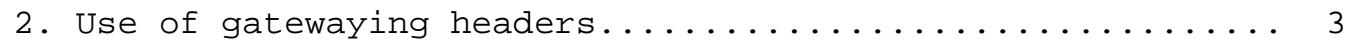

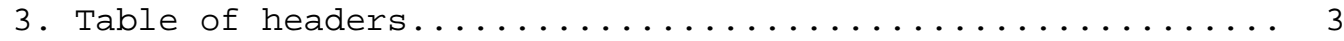

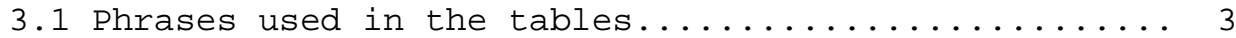

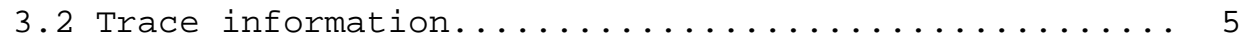

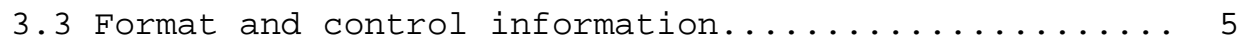

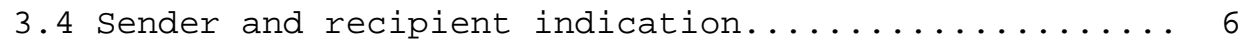

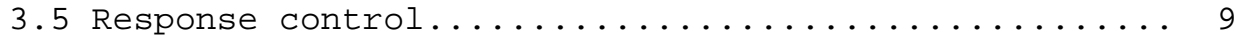

3.6 Message identification and referral headers....... 11

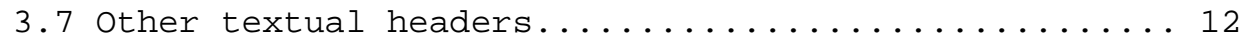

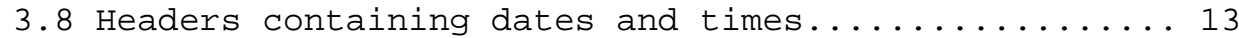

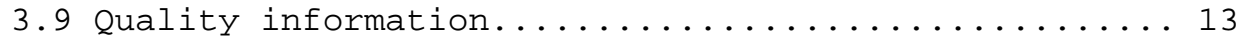

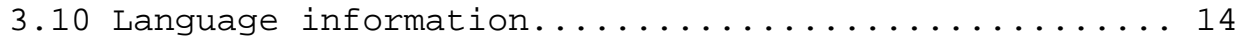

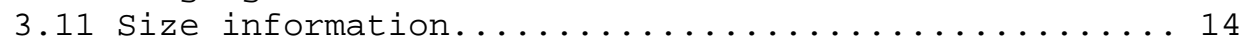

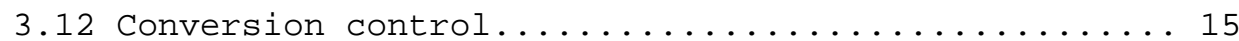

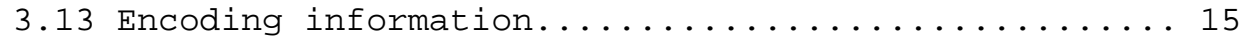

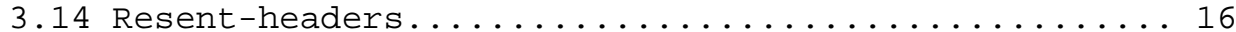

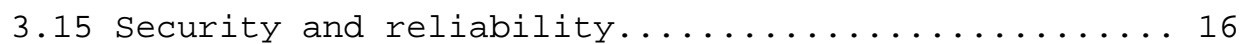

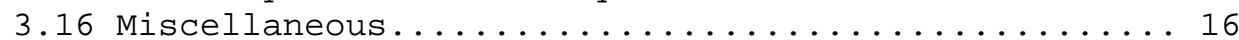

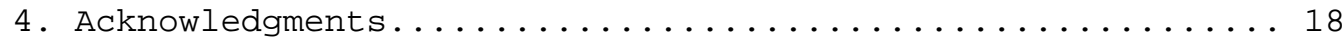




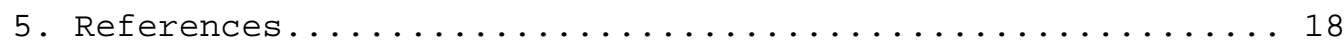

6. Author's Address........................... 20

Appendix A:

Headers sorted by Internet RFC document in which they appear. 21 Appendix B:

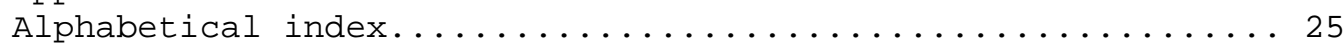

1. Introduction

Many different Internet standards and RFCs define headers which may occur on Internet Mail Messages and Usenet News Articles. The intention of this document is to list all such headers in one document as an aid to people developing message systems or interested in Internet Mail standards.

The document contains all headers which the author has found in the following Internet standards: , RFC 822 [2], RFC 1036 [3], RFC 1123 [5], $\operatorname{RFC} 1327$ [7], $\mathrm{RFC} 1496$ [8], $\mathrm{RFC} 1521$ [11], RFC 1766 [12], RFC 1806 [14], RFC 1864[17] and RFC 1911[20]. Note in particular that heading attributes defined in PEM (RFC 1421-1424) and MOSS (RFC 1848 [16]) are not included. PEM and MOSS headers only appear inside the body of a message, and thus are not headers in the RFC 822 sense. Mail attributes in envelopes, i.e. attributes controlling the message transport mechanism between mail and news servers, are not included. This means that attributes from SMTP [1], UUCP [18] and NNTP [15] are mainly not covered either. Headings used only in HTTP [19] are not included yet, but may be included in future version of this memo. A few additional headers which often can be found in e-mail headings but are not part of any Internet standard are also included.

For each header, the document gives a short description and a reference to the Internet standard or RFC, in which they are defined.

The header names given here are spelled the same way as when they are actually used. This is usually American but sometimes English spelling. One header in particular, "Organisation/Organization", occurs in e-mail headers sometimes with the English and other times with the American spelling.

The following words are used in this memo with the meaning specified below:

heading Formatted text at the top of a message, ended by a blank line

header $=$ heading One field in the heading, beginning with a field field name, colon, and followed by the field value(s) 
It is my intention to continue updating this document after its publication as an RFC. The latest version, which may be more up-todate (but also less fully checked out) will be kept available for downloading from URL

http://www.dsv.su.se/ jpalme/ietf-mail-attributes.pdf .

Please e-mail me (Jacob Palme <jpalmeddsv.su.se>) if you have noted headers which should be included in this memo but are not.

2. Use of gatewaying headers

RFC 1327 defines a number of new headers in Internet mail, which are defined to map headers which X.400 has but which were previously not standardized in Internet mail. The fact that a header occurs in RFC 1327 indicates that it is recommended for use in gatewaying messages between X.400 and Internet mail, but does not mean that the header is recommended for messages wholly within Internet mail. Some of these headers may eventually see widespread implementation and use in Internet mail, but at the time of this writing (1996) they are not widely implemented or used.

Headers defined only in RFC 1036 for use in Usenet News sometimes appear in mail messages, either because the messages have been gatewayed from Usenet News to e-mail, or because the messages were written in combined clients supporting both e-mail and Usenet News in the same client. These headers are not standardized for use in Internet e-mail and should be handled with caution by e-mail agents.

3. Table of headers

3.1 Phrases used in the tables

"not for general usage"
Used to mark headers which are defined in RFC 1327 for use in messages from or to Internet mail/X.400 gateways. These headers have not been standardized for general usage in the exchange of messages between Internet mailbased systems. 
"not standardized for use in e-mail"

"non-standard"

"discouraged"

"controversial"

"experimental"
Used to mark headers defined only in RFC 1036 for use in Usenet News. These headers have no standard meaning when appearing in e-mail, some of them may even be used in different ways by different software. When appearing in e-mail, they should be handled with caution. Note that RFC 1036, although generally used as a de-facto standard for Usenet News, is not an official IETF standard or even on the IETF standards track.

This header is not specified in any of referenced RFCs which define Internet protocols, including Internet Standards, draft standards or proposed standards. The header appears here because it often appears in email or Usenet News. Usage of these headers is not in general recommended. Some header proposed in ongoing IETF standards development work, but not yet accepted, are also marked in this way.

This header, which is non-standard, is known to create problems and should not be generated. Handling of such headers in incoming mail should be done with great caution.

The meaning and usage of this header is controversial, i.e. different implementors have chosen to implement the header in different ways. Because of this, such headers should be handled with caution and understanding of the different possible interpretations.

This header is used for newly defined headers, which are to be tried out before entering the IETF standards track. These should only be used if both communicating parties agree on using them. In practice, some experimental protocols become de-facto-standards before they are made into IETF standards. 


\subsection{Trace information}

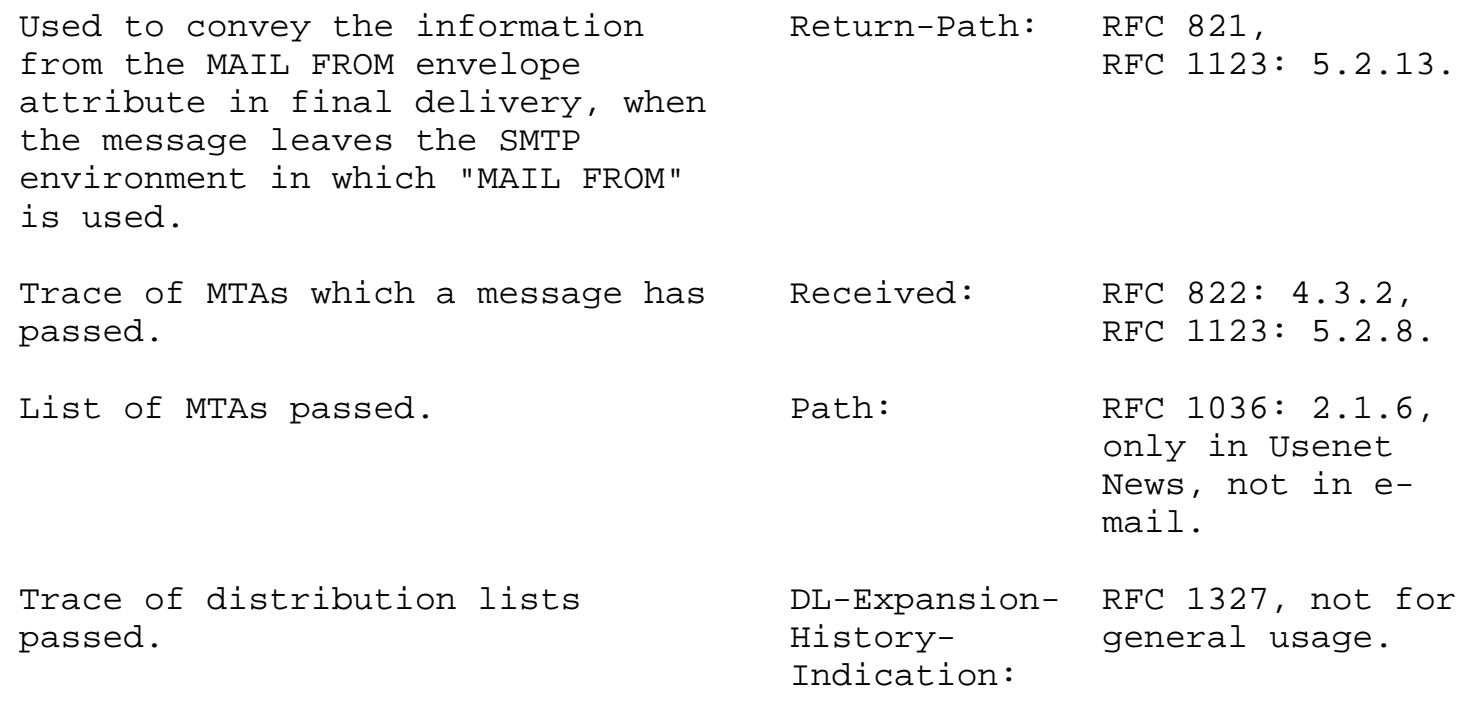

3.3 Format and control information

An indicator that this message is

MIME-Version: RFC 1521: 3 . formatted according to the MIME standard, and an indication of which version of MIME is utilized.

Special Usenet News actions only.

Control:

RFC 1036: 2.1.6, only in Usenet News, not in email.

Special Usenet News actions and a normal article at the same time.

Also-Control:

son-of-RFC1036

[21], nonstandard, only in Usenet News, not in e-mail

Which body part types occur in OriginalEncodedInformationTypes:

RFC 1327, not for general usage. 
Controls whether this message may be forwarded to alternate recipients such as a postmaster if delivery is not possible to the intended recipient. Default: Allowed.

Whether recipients are to be told the names of other recipients of the same message. This is primarily an X.400 facility. In $\mathrm{X} .400$, this is an envelope attribute and refers to disclosure of the envelope recipient list. Disclosure of other recipients is in Internet mail done via the To:, cc: and bcc: headers.

Whether a MIME body part is to be shown inline or is an attachment; can also indicate a suggested filename for use when saving an attachment to a file.

3.4 Sender and recipient indication

Authors or persons taking responsibility for the message.

Note difference from the "From " header (not followed by ":") below.

(1) This header should never appear in e-mail being sent, and should thus not appear in this memo. It is however included, since people often ask about it.
AlternateRecipient:

DiscloseRecipients:
RFC 1327, not for general usage.

RFC 1327, not for general usage.
Content-
Disposition:

RFC 1806,

experimental
From:

From
RFC 822: 4.4.1, $\mathrm{RFC} 1123$ : 5.2.15$16,5.3 .7$, RFC 1036 2.1.1

not standardized for use in e-mail 
This header is used in the socalled Unix mailbox format, also known as Berkely mailbox format or the MBOX format. This is a format for storing a set of messages in a file. A line beginning with "From " is used to separate successive messages in such files.

This header will thus appear when you use a text editor to look at a file in the Unix mailbox format. Some mailers also use this format when printing messages on paper.

The information in this header should NOT be used to find an address to which replies to a message are to be sent.

(2) Used in Usenet News mail transport, to indicate the path through which an article has gone when transferred to a new host.

Sometimes called "From_" header.

Name of the moderator of the newsgroup to which this article is sent; necessary on an article sent to a moderated newsgroup to allow its distribution to the newsgroup members. Also used on certain control messages, which are only performed if they are marked as Approved.

The person or agent submitting the message to the network, if other than shown by the From: header.

Primary recipients.
From

or

$>$ From

Approved:

Sender:

To:
RFC 976: 2.4 for use in Usenet News
RFC 1036: 2.2.11, not standardized for use in e-mail. 


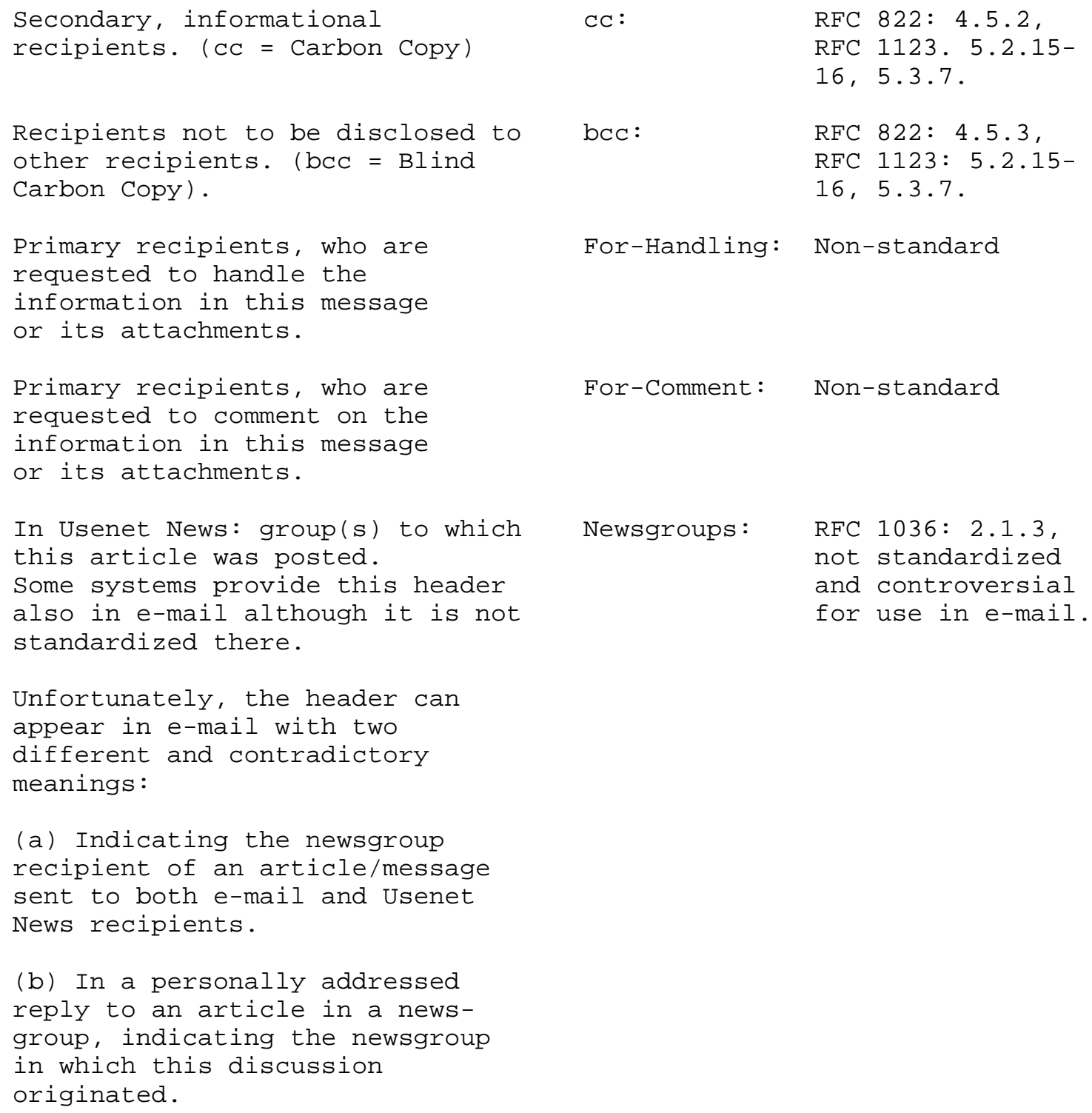




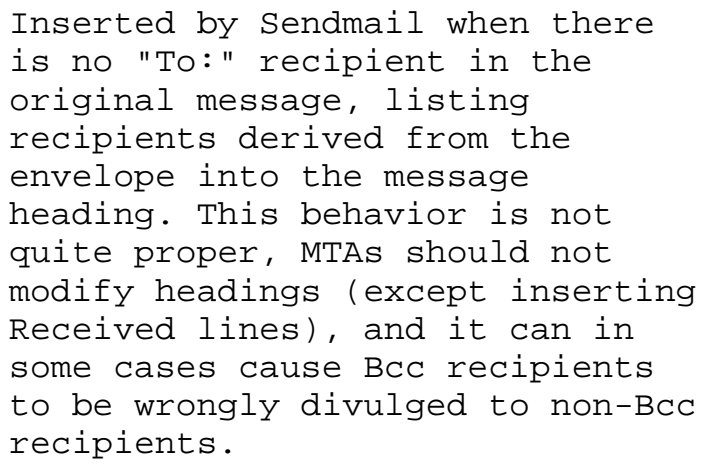

3.5 Response control

This header is meant to indicate where the sender wants replies to go. Unfortunately, this is ambiguous, since there are different kinds of replies, which the sender may wish to go to different addresses. In particular, there are personal replies intended for only one person, and group replies, intended for the whole group of people who read the replied-to message (often a mailing list, anewsgroup name cannot appear here because of different syntax, see "Followup-To" below.).

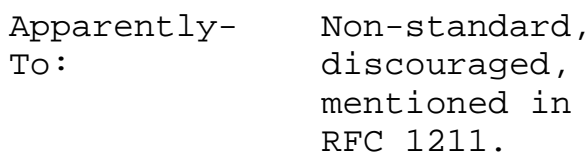

Distribution:

RFC 1036: 2.2.7, not standardized for use in e-mail.

Fax:, Telefax:

Non-standard.

Phone:

Non-standard.

Mail-SystemVersion:, Mailer:, OriginatingClient:, $\mathrm{X}-$ Mailer, $\mathrm{X}-$ Newsreader

Reply-To:

RFC 822: 4.4.3, RFC 1036: 2.2.1 controversial. 
Some mail systems use this header to indicate a better form of the e-mail address of the sender. Some mailing list expanders puts the name of the list in this header. These practices are controversial. The personal opinion of the author of this RFC is that this header should be avoided except in special cases, but this is a personal opinion not shared by all specialists in the area.

Used in Usenet News to indicate that future discussions (=followup) on an article should go to a different set of newsgroups than the replied-to article. The most common usage is when an article is posted to several newsgroups, and further discussions is to take place in only one of them.

In e-mail, this header may occur in a message which is sent to both e-mail and Usenet News, to show where follow-up in Usenet news is wanted. The header does not say anything about where follow-up in e-mail is to be sent.

Note that the value of this header must always be one or more newsgroup names, never e-mail addresses.

Address to which notifications are to be sent and a request to get delivery notifications. Internet standards recommend, however, the use of RCPT TO and Return-Path, not Errors-To, for where delivery notifications are to be sent.
Followup-To: RFC 1036: 2.2.3, not standardized for use in e-mail. 
Whether non-delivery report is wanted at delivery error. Default is to want such a report.

Whether a delivery report is wanted at successful delivery. Default is not to generate such a report.

Indicates whether the content of a message is to be returned with non-delivery notifications.

Possible future change of name for "Content-Return:"

3.6 Message identification and referral headers

Unique ID of this message.

Unique ID of one body part of the content of a message.

Base to be used for resolving relative URIs within this content part.

URI with which the content of this content part might be retrievable.

Reference to message which this message is a reply to.

In e-mail: reference to other related messages, in Usenet News: reference to replied-to-articles.

References to other related articles in Usenet News.

Reference to previous message being corrected and replaced. Compare to "Supersedes:" below. This field may in the future be replaced with "Supersedes:".
PreventNonDeliveryReport:

GenerateDeliveryReport:

ContentReturn:

X400-ContentReturn:
RFC 1327, not for general usage.

RFC 1327, not for general usage.

RFC 1327, not for general usage.

non-standard 
Commonly used in Usenet News in similar ways to the "Obsoletes" header described above. In Usenet News, however, Supersedes causes a full deletion of the replaced article in the server, while "Supersedes" and "Obsoletes" in email is implemented in the client and often does not remove the old version of the text.

Only in Usenet News, similar to "Supersedes:" but does not cause the referenced article to be physically deleted.

Reference to specially important articles for a particular Usenet Newsgroup.

3.7 Other textual headers

Search keys for data base retrieval.

Title, heading, subject. Often used as thread indicator for messages replying to or commenting on other messages.

Comments on a message.

Description of a particular body part of a message.

Organization to which the sender of this article belongs.

See Organization above.

Short text describing a longer article. Warning: Some mail systems will not display this text to the recipient. Because of this, do not use this header for text which you want to ensure that the recipient gets.
Supersedes: $\quad$ son-of-RFC1036

[21], non-standard

Article-

son-of-RFC1036

Updates :

[21], non-standard

Article-

son-of-RFC1036

Names:

[21], non-standard 
A text string which identifies

the content of a message.

3. 8 Headers containing dates and times

The time when a message was

delivered to its recipient.

In Internet, the date when a message was written, in X.400, the time a message was submitted. Some Internet mail systems also use the date when the message was submitted.

A suggested expiration date. Can be used both to limit the time of an article which is not meaningful after a certain date, and to extend the storage of important articles.

Time at which a message loses its validity. This field may in the future be replaced by "Expires:"

Latest time at which a reply is requested (not demanded).

\subsection{Quality information}

Can be "normal", "urgent" or "nonurgent" and can influence transmission speed and delivery.

Sometimes used as a priority value which can influence transmission speed and delivery. Common values are "bulk" and "first-class". Other uses is to control automatic replies and to control return-of-content facilities, and to stop mailing list loops.

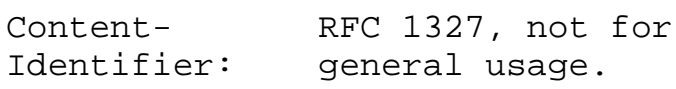

DeliveryDate:

Date:

Expires:

Expiry-Date:

RFC 1327, not for general usage.

Reply-By :

RFC 1327, not for general usage.

Priority:

RFC 1327, not for general usage.

Precedence: Non-standard, controversial, discouraged. 
A hint from the originator to the recipients about how important a message is. Values: High, normal or low. Not used to control transmission speed.

How sensitive it is to disclose this message to other people than the specified recipients. Values: Personal, private, company confidential. The absence of this header in messages gatewayed from $\mathrm{X} .400$ indicates that the message is not sensitive.

Body parts are missing.

\subsection{Language information}

Can include a code for the natural language used in a message, e.g. "en" for English.

Can include a code for the natural language used in a message, e.g. "en" for English.

\subsection{Size information}

Inserted by certain mailers to indicate the size in bytes of the message text. This is part of a format some mailers use when showing a message to its users, and this header should not be used when sending a message through the net. The use of this header in transmission of a message can cause several robustness and interoperability problems.

Size of the message.

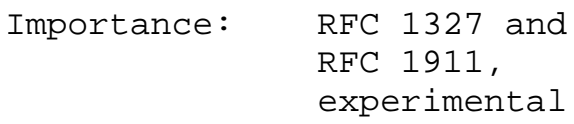

IncompleteCopy :

RFC 1327, not for general usage.

Language:

ContentLanguage:

RFC 1327, not for general usage.

RFC 1766, proposed standard.

ContentNon-standard, Length: discouraged. 


\subsection{Conversion control}

The body of this message may not be converted from one character set to another. Values: Prohibited and allowed.

Non-standard variant of Conversion: with the same values.

The body of this message may not be converted from one character set to another if information will be lost. Values: Prohibited and allowed.

\subsection{Encoding information}

Format of content (character set etc.) Note that the values for this header are defined in different ways in RFC 1049 and in MIME (RFC 1521), look for the "MIME-version" header to understand if Content-Type is to be interpreted according to RFC 1049 or according to MIME. The MIME definition should be used in generating mail.

RFC 1766 defines a parameter "difference" to this header. Information from the SGML entity declaration corresponding to the entity contained in the body of the body part.

Coding method used in a MIME message body .

Only used with the value "Delivery Report" to indicates that this is a delivery report gatewayed from X.400.

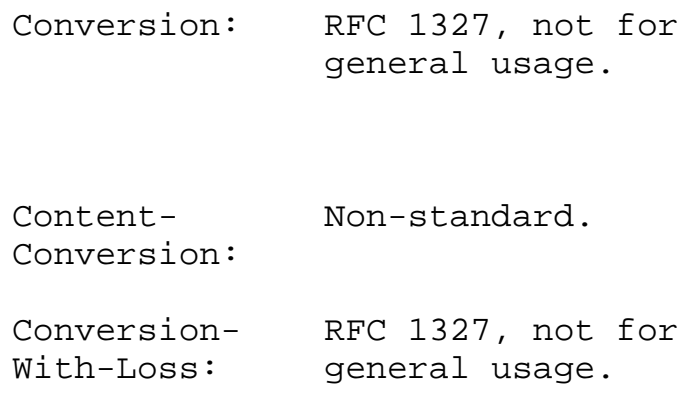

Non-standard.

.

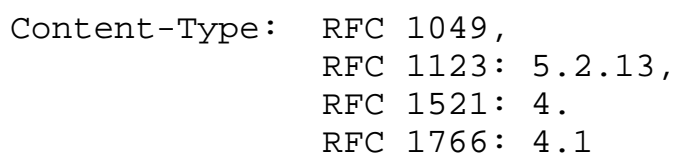


Used in several different ways by different mail systems. Some use it for a kind of content-type information, some for encoding and length information, some for a kind of boundary information, some in other ways.

\subsection{Resent-headers}

When manually forwarding a message, headers referring to the forwarding, not to the original message. Note: MIME specifies another way of resending messages, using the "Message" Content-Type.

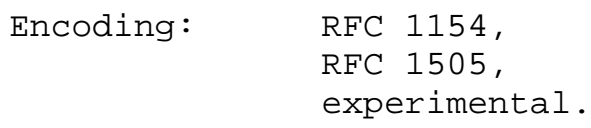

3.15 Security and reliability

Checksum of content to ensure that it has not been modified.

Used in Usenet News to store information to avoid showing a reader the same article twice if it was sent to more than one newsgroup. Only for local usage within one Usenet News server, should not be sent between servers.

\subsection{Miscellaneous}

Name of file in which a copy of

FCC :

Non-standard.

Has been automatically forwarded.

Auto-

Forwarded:
RFC 1864, proposed standard.

RFC 1036: 2.2.13, only in Usenet News, not in email. 


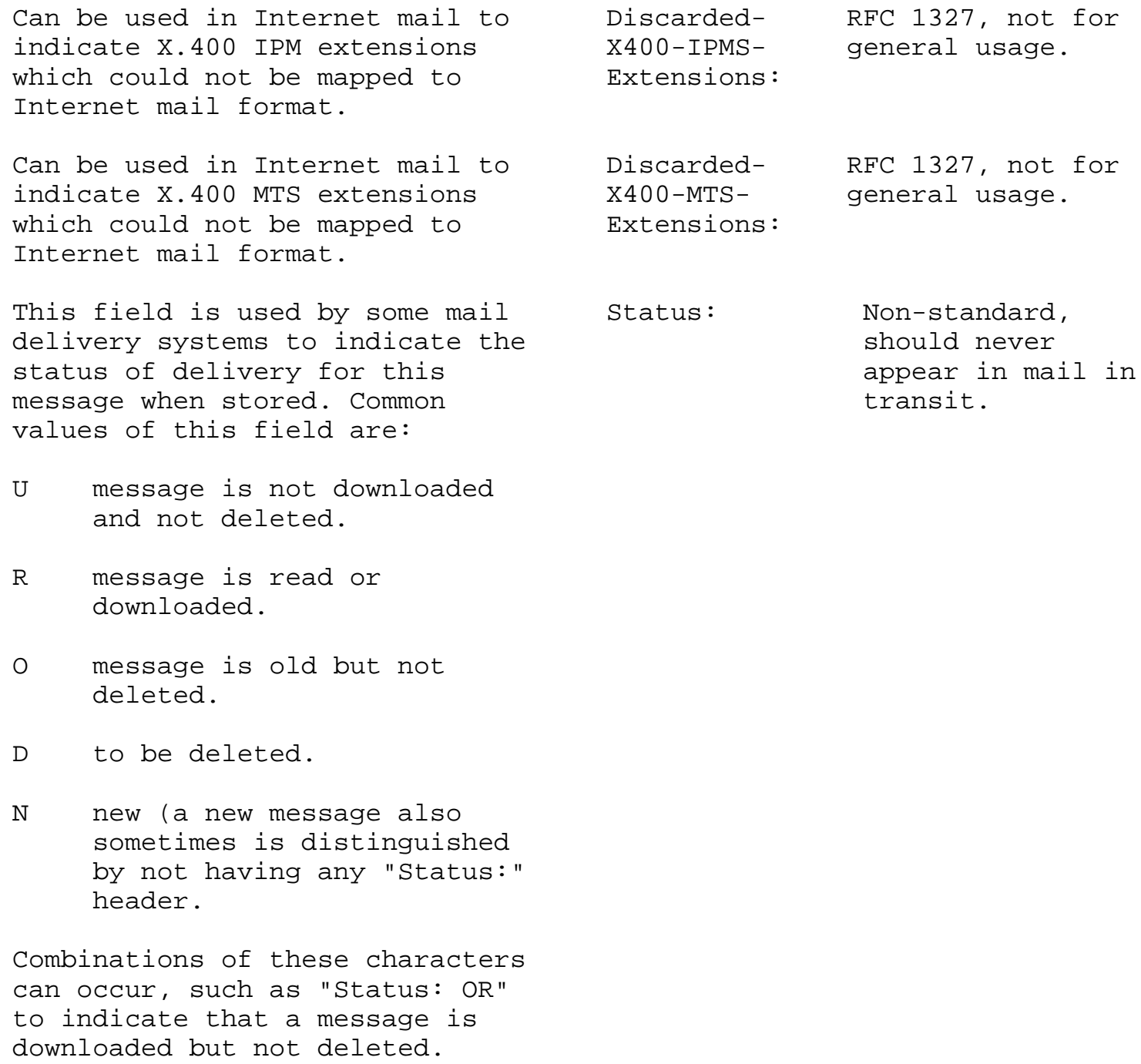

DiscardedX400-IPMSindicate X.400 IPM extensions which could not be mapped to Internet mail format.

Can be used in Internet mail to indicate X.400 MTS extensions which could not be mapped to Internet mail format.

This field is used by some mail delivery systems to indicate the status of delivery for this message when stored. Common values of this field are:

$\mathrm{U}$ message is not downloaded and not deleted.

$R$ message is read or downloaded.

O message is old but not deleted.

D to be deleted.

$\mathrm{N}$ new (a new message also sometimes is distinguished by not having any "Status:" header.

Combinations of these characters can occur, such as "Status: OR" to indicate that a message is downloaded but not deleted.

RFC 1327, not for general usage.

Extensions:

DiscardedX400-MTS-

Extensions:

RFC 1327, not for general usage.

Status: should never appear in mail in transit. 


\section{Acknowledgments}

Harald Tveit Alvestrand, Ned Freed, Olle Jdrnefors, Keith Moore, Nick Smith and several other people have helped me with compiling this list. I especially thank Ned Freed and Olle Jdrnefors for their thorough review and many helpful suggestions for improvements. I alone take responsibility for any errors which may still be in the list.

An earlier version of this list has been published as part of [13].

5. References

Ref. Author, title

IETF status

( July 1996)

[1] J. Postel: "Simple Mail Transfer Protocol", STD 10, RFC 821, August 1982.

Standard, Recommended

[2] D. Crocker: "Standard for the format of ARPA Internet text messages." STD 11, RFC 822, August 1982 .

[3] M.R. Horton, R. Adams: "Standard for interchange of USENET messages", RFC 1036, December 1987 .

[4] M. Sirbu: "A Content-Type header header for internet messages", RFC 1049, March 1988.

[5] R. Braden (editor): "Requirements for Internet Hosts -- Application and Support", STD-3, RFC 1123, October 1989.

Standard, Recommended

Not an official IETF standard, but in reality a defacto standard for Usenet News

Standard, Recommended, but can in the future be expected to be replaced by MIME

Standard, Required

[6] D. Robinson, R. Ullman: "Encoding Header Header for Internet Messages", RFC 1154, Non-standard April 1990 . 
[7] S. Hardcastle-Kille: "Mapping between X.400(1988) / ISO 10021 and RFC 822", RFC 1327 May 1992.

[8] H. Alvestrand \& J. Romaguera: "Rules for Downgrading Messages from X.400/88 to X.400/84 When MIME Content-Types are Present in the Messages", RFC 1496, August 1993.

[9] A. Costanzo: "Encoding Header Header for Internet Messages", RFC 1154, April 1990.

[10] A. Costanzo, D. Robinson: "Encoding Header Header for Internet Messages", RFC 1505, August 1993 .

[16] 1848 PS S. Crocker, N. Freed, J. Galvin, S. Murphy, "MIME Object Security Services", RFC 1848, March 1995.

[17] J. Myers, M. Rose: The Content-MD5 Header Header, RFC 1864, October 1995.

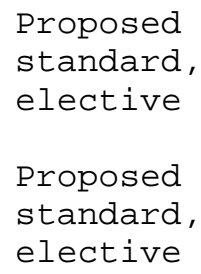

Non-standard

Experimental

Draft

Standard, elective

Proposed standard, elective

Non-standard

Experimental

Proposed standard

Proposed standard

Draft standard 
M. Horton, UUCP mail interchange format standard, RFC 976, Januari 1986.

[19]

T. Berners-Lee, R. Headering, H. Frystyk: Hypertext Transfer Protocol -- HTTP/1.0, RFC 1945, May 1996.

[20] G. Vaudreuil: Voice Profile for Internet Mail, RFC 1911, February 1996.

[21] H. Spencer: News Article Format and Transmission, June 1994, FTP://zoo.toronto.edu/pub/news.ps FTP://zoo.toronto.edu/pub/news.txt.z

This document is often referenced under the name "son-of-RFC1036".

\author{
Not an offi- \\ cial IETF \\ standard, \\ but in \\ reality a de- \\ facto \\ standard for \\ Usenet News \\ Not an official \\ IETF standard, \\ but the defacto \\ standard until \\ the next \\ version is \\ published \\ Experimental
Not even an
$\mathrm{RFC}$, but
still widely
used and
partly
almost a de-
facto
standard for
Usenet News

6. Author's Address

Jacob Palme
Stockholm University/KTH
Electrum 230
S-164 40 Kista, Sweden
Phone: +46-8-1616 67

Fax: +46-8-783 0829

E-mail: jpalmeddsv.su.se 
Appendix A:

Headers sorted by Internet RFC document in which they appear.

RFC 822

$\mathrm{bcc}$

$\mathrm{CC}$

Comments

Date

From

In-Reply-To

Keywords

Message-ID

Received

References

Reply-To

Resent-

Resent-bcc

Resent-cC

Resent-Date

Resent-From

Resent-From

Resent-Message-ID

Resent-Reply-To

Resent-To

Return-Path

Sender

Sender

Subject

To

RFC 976

-------

"From " (followed by space, not colon (:") 
RFC 1036

--------

Approved

Control

Distribution

Expires

Followup-To

Lines

Newsgroups

Organization

Path

Summary

Xref

RFC 1049

--------

Content-Type

RFC 1327

Alternate-recipient

Auto-Forwarded

Autoforwarded

Content-Identifier

Content-Return

Conversion

Conversion-With-Loss

Delivery-Date

Discarded-X400-IPMS-Extensions

Discarded-X400-MTS-Extensions

Disclose-Recipients

DL-Expansion-History

Expiry-Date

Generate-Delivery-Report

Importance

Incomplete-Copy

Language

Message-Type Delivery

Obsoletes

Original-Encoded-Information-Types

Prevent-NonDelivery-Report

Priority

Reply-By

Report

Sensitivity 
RFC 1505

--------

Encoding

RFC 1521

--------

Content-Description

Content-ID

Content-Transfer-Encoding

Content-Type

MIME-Version

RFC 1806

$-------$

Content-Disposition

RFC 1864

$--------$

Content-MD 5

RFC 1911

Importance

Sensitivity

son-of-RFC1036 [21]

-------------------

Also-Control

Article-Names

Article-Updates

See-Also

Supersedes 
Not Internet standard

Apparently-to

Content-Base

Content-Length

Content-Location

Content-SGML-Entity

Encoding

Errors-To

Return-Receipt-To

Fax

"From " (not followed by ":")

Telefax

$\mathrm{FCC}$

For-Comment

For-Handling

Mail-System-Version

Mailer

Organisation

Originating-Client

Phone

Status

Supersedes

X400-Content-Return

$\mathrm{X}$-Mailer

$\mathrm{X}$-Newsreader 
Appendix B:

Alphabetical index

Section Heading-header

3.3 Also-Control

3.3 Alternate-Recipient

3.4 Apparently-To

3.4 Approved

3.6 Article-Names

3.6 Article-Updates

3.16 Auto-Forwarded

$3.4 \quad \mathrm{bcc}$

$3.4 \quad \mathrm{cC}$

Client, see Originating-Client

3.7 Comments

3.6 Content-Base

3.12 Content-Conversion

3.7 Content-Description

3.3 Content-Disposition

3.6 Content-ID

3.7 Content-Identifier

3.10 Content-Language see also Language

3.11 Content-Length

3.6 Content-Location

3.15 Content-MD5

3.4 Content-Return

3.13 Content-SGML-Entity

3.13 Content-Transfer-Encoding

3.13 Content-Type

3.3 Control

3.12 Conversion

3.12 Conversion-With-Loss

3.8 Date

3.8 Delivery-Date

Delivery-Report, see Generate-Delivery-Report, PreventDelivery-Report, Non-Delivery-Report, Content-Type

Description, see Content-Description

3.16 Discarded-X400-IPMS-Extensions

3.16 Discarded-X400-MTS-Extensions

3.3 Disclose-Recipients

3.4 Disposition, see Content-Disposition

3.4 Distribution

3.2 DL-Expansion-History-Indication

3.13 Encoding see also Content-Transfer-Encoding

3.4 Errors-To 


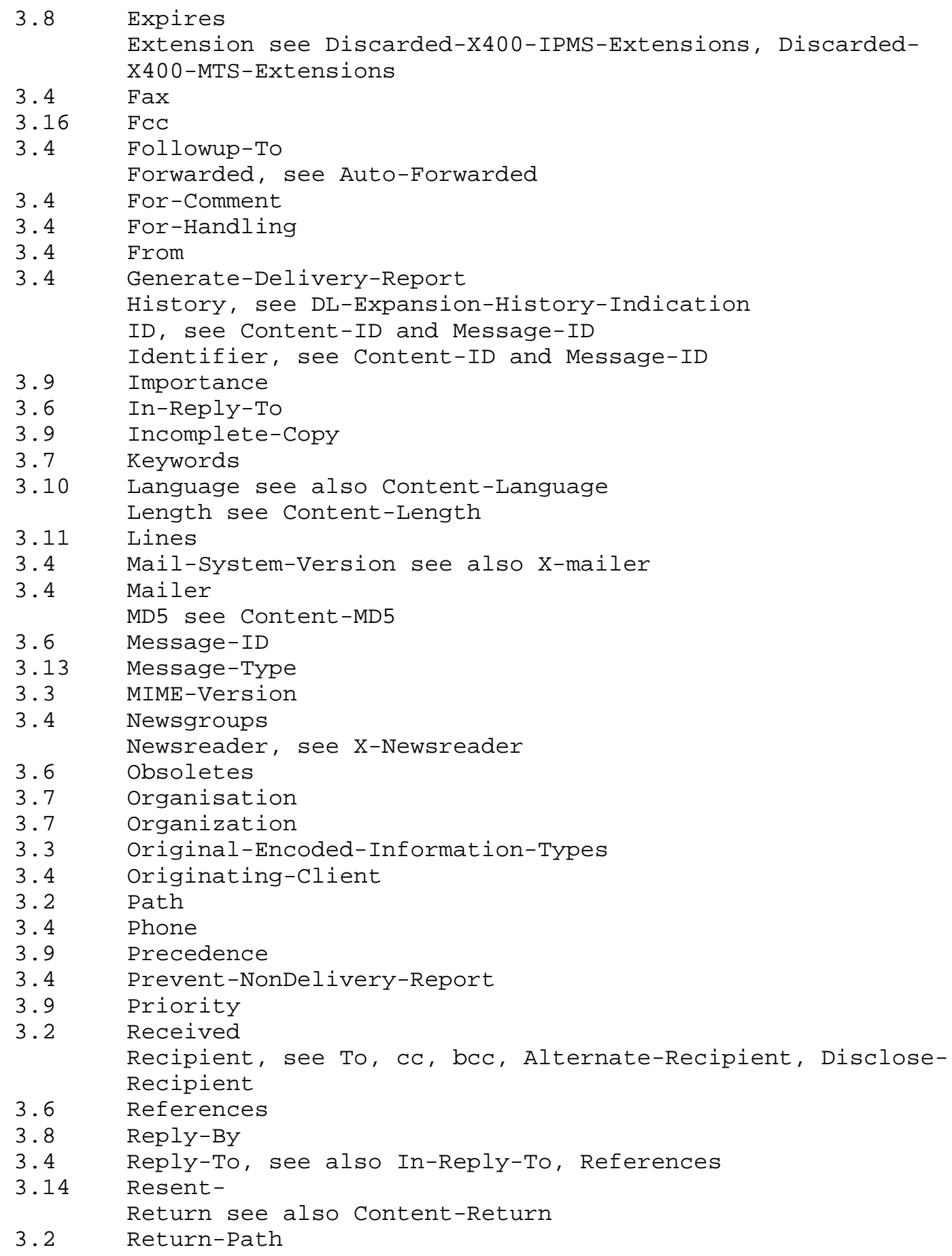




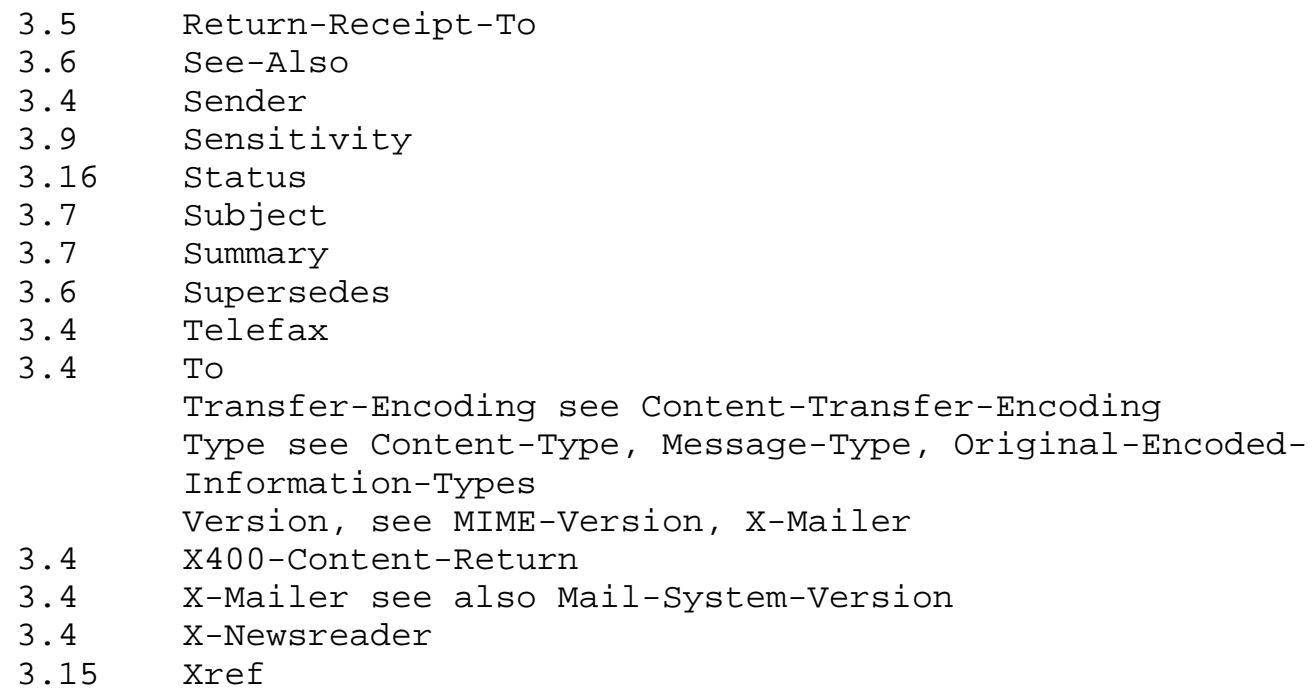

